\title{
VLF Propagation Under the Ionosphere in the Lowest Mode of Horizontal Polarization
}

\author{
Harold A. Wheeler
}

\author{
Contribution From the Wheeler Laboratories, Great Neck, N.Y.
}

\begin{abstract}
In the lower part of the VLF band, around $4 \mathrm{kc} / \mathrm{s}$, it appears that the lowest rate of attenuation is obtainable by horizontal polarization in the TE-01 mode. This offers a substantial advantage relative to vertical polarization in the usual TM-01 mode and the simple TM-00 or TEM mode. Some types of antennas are found to be suitable for the TE-01 mode, namely, a horizontal wire above ground or a vertical loop, either one located in a plane perpendicular to the direction of transmission. A theoretical study is summarized, leading to the conclusion that this mode offers some unique features and is suitable for transmission to distances of the order of $4,000 \mathrm{~km}$.
\end{abstract}

\section{Introduction}

The practical utilization of the VLF band has been limited to vertical polarization, and the same is true of most theoretical studies. It now appears that there is a part of the frequency spectrum which would be best utilized by the use of horizontal polarization.

Practical utilization of the VLF band ( 3 to $30 \mathrm{kc} / \mathrm{s}$ ) has been limited to the upper part $(10$ to $30 \mathrm{kc} / \mathrm{s})$ where admittedly the most efficient transmission under the ionosphere appears to be obtained by vertical polarization. The prospects for the lower part (3 to $10 \mathrm{kc} / \mathrm{s}$ ) have been dimmed by the observation of maximum attenuation of noise (at 3 to $4 \mathrm{kc} / \mathrm{s}$ ) but it has not been sufficiently emphasized that this handicap applies only to vertical polarization.

The appreciation of this fact about 4 years ago encouraged the writer to consider the alternative of horizontal polarization for utilization of frequencies in the lower part of the VLF band (say around 4 $\mathrm{kc} / \mathrm{s}$ ). The resulting theoretical studies indicate the utility of this part of the spectrum is best utilized by horizontal polarization.

It is found that a single mode of $\mathrm{HP}$, the TE-01 mode, promises the lowest rate of attenuation of all modes, in a limited range of the spectrum. The principal advantage is the more effective reflection from the ionosphere. Another advantage is the use of a single mode as distinguished from a mixture of the two principal modes of vertical polarization, the usual TM-01 and the simple TM-00 or TEM.

The use of a different mode naturally introduces opportunities and problems that are challenging. These will be presented and summarized in support of the thesis. The essential characteristics of different types of antennas will be compared. An example (computed for $4 \mathrm{kc} / \mathrm{s}$ ) indicates that useful transmission is possible to distances of the order of $4,000 \mathrm{~km}$.

\section{Theory of Propagation}

Propagation with horizontal polarization under the ionosphere has some major differences from vertical polarization, in regard to its behavior and limitations. Therefore it is essential to have a clear view of these distinctions.

In order to present the subject in proper prespective, it will be necessary to compare the horizontally polarized mode with its closest competitors, the lowest two of the vertically polarized modes. These different modes are designated as follows:

Vertical polarization,

Vertical polarization,

Horizontal polarization,

These designations follow the pattern of mode numbers in a rectangular waveguide. The first digit (0) denotes uniform field across the width, which results from the absence of side walls. The second digit (0 or 1$)$ denotes the number of "half-wave" cycles of field variation in the height of the waveguide. There is a recognizable correlation between these designations and the same or similar ones in use by other writers.

Figure 1 shows the field pattern of the subject mode, TE-01, characterized by horizontal polarization of the electric field $(E)$. The upper and lower boundaries of the waveguide are the ionosphere and the ground (land or water), separated by the effective height $(h)$. The magnetic field $(H)$ has a vertical component and also has a component in the direction of propagation, which are related to the concept of two component waves with oblique propagation. Each "half-wave" cycle of the field pattern is associated with a half-wavelength in the guide $\left(1 / 2 \lambda_{g}\right)$.

The principal discussion of the waveguide behavior will be based on magnetic-dipole (loop) antennas at the sending and receiving ends $(S$ and $R$ ). The vertical loop is the one kind of antenna which is 


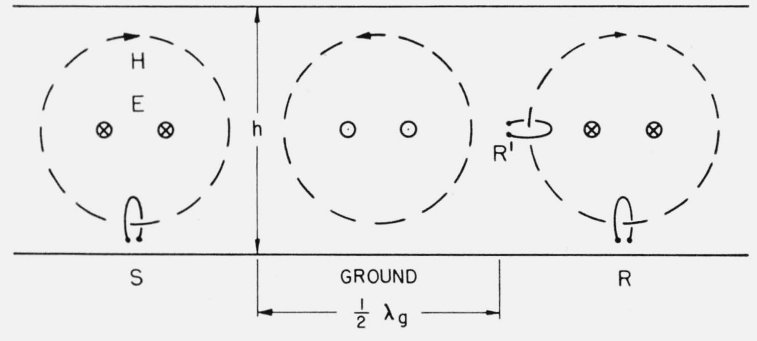

TE-OI MODE ( HORIZONTAL POLARIZATION)

FIguRE 1. Wave propagation in TE-01 mode under ionosphere.

adapted to all three modes. Its operation in "vertical polarization" is well known, in that the transverse magnetic field (TM) is horizontal. Its operation in horizontal polarization is unusual, in that the horizontal longitudinal component of magnetic field is the principal component near the boundaries (ionosphere and ground). Therefore the loop axis is along the line of propagation, a direction which gives no radiation in free space. Here it does couple with the waveguide mode. This may be explained by its substantial radiation in the oblique directions of propagation of the pair of component waves in the guide.

Figure 1 shows how vertical loops in coaxial relation may be used on the ground for sending and receiving ( $S$ and $R$ ). On aircraft at a substantial altitude, the horizontal component of magnetic field decreases while the vertical component increases (respectively reaching zero and maixmum at $1 / 2 h$ ). Therefore a horizontal loop on an aircraft may serve as a receiving antenna $\left(R^{\prime}\right)$.

By comparison with figure 1, the vertically polarized modes have different patterns with only horizontal magnetic field. The TM-00 or TEM mode has uniform vertical electric field terminating on the boundaries. The TM-01 mode has a vertical component of electric field of maximum value but opposite polarity at the upper and lower boundaries. Also it has a horizontal component in the direction of propagation, which is not usually utilized.

For this study, the following ionosphere conditions are assumed; they are believed to be typical of the actual range of conditions:

Effective height of ionosphere:

Cutoff wavelength of 01 modes:

Cutoff frequency:

$$
h=75 \mathrm{~km} \text {. }
$$

$\lambda_{c}=2 h=150 \mathrm{~km}$.

$f_{c}=2 \mathrm{kc} / \mathrm{s}$.

These conditions are based on the concept that the boundaries behave as fairly good conductors.

This concept fails for the TM-01 mode under usual VLF operating conditions (10 to $30 \mathrm{kc} / \mathrm{s})$; this will be taken into account in drawing conclusions. Under the extreme conditions of low conductivity and high frequency, the upper boundary behaves more like a magnetic wall (open-circuit instead of short-circuit), the phase of reflection being reversed. This concept would lead to a mode designated TM-
$0 \frac{1}{2}$ and a nominal cutoff frequency of $1 \mathrm{kc} / \mathrm{s}$. However, the latter is not realized, because this concept is not applicable near cutoff.

As mentioned above, the waveguide behavior can be explained in terms of a pair of oblique waves reflected from the boundaries. Figure 2 shows this familiar concept as applied to the present subject, particularly the TE-01 and TM-01 modes. The sending antenna, with its images in the boundaries, forms a pair of waves that are incident on the boundaries at an elevation angle $(\psi)$ from parallel (grazing incidence). These waves undergo reflection at the boundaries. At the ionosphere, the reflection coefficient $(\rho)$ is less than unity and this deficiency causes a loss in propagation out to the transmission radius $(r)$. Corresponding loss in the ground reflection (not shown) is much less and will be ignored. The propagation loss may be computed from the reflection loss multiplied by the number of reflections.

The ionosphere may be described in terms of its conductivity $(\sigma)$ and some resulting derived factors, as follows:

$$
\begin{aligned}
& \text { Dissipation factor: } p=\frac{\sigma}{\omega \epsilon}=2\left(\frac{\lambda}{2 \pi \delta}\right)^{2} \\
& \text { Skin depth: } \delta=\sqrt{\frac{\lambda}{\pi \sigma R_{c}} ;} \\
& \qquad \frac{2 \pi \delta}{\lambda}=\sqrt{\frac{4 \pi}{\lambda \sigma R_{c}}}=\sqrt{\frac{1}{30 \sigma \lambda}}=\sqrt{2 / p}
\end{aligned}
$$

in which

$\sigma=$ conductivity, uniform above a certain level of altitude (mhos/meter).

$\omega=2 \pi f=$ radian frequency (radians/second).

$\epsilon=8.85 \times 10^{-12}=$ electrivity (electric permittivity) in free space, including ionosphere (farads/meter).

$\lambda=$ free-space wavelength corresponding to the operating frequency (meters).

$R_{c}=377=$ free-space wave resistance across a square area of wave front (ohms).

$\delta=$ skin depth on surface of conductor (meters).

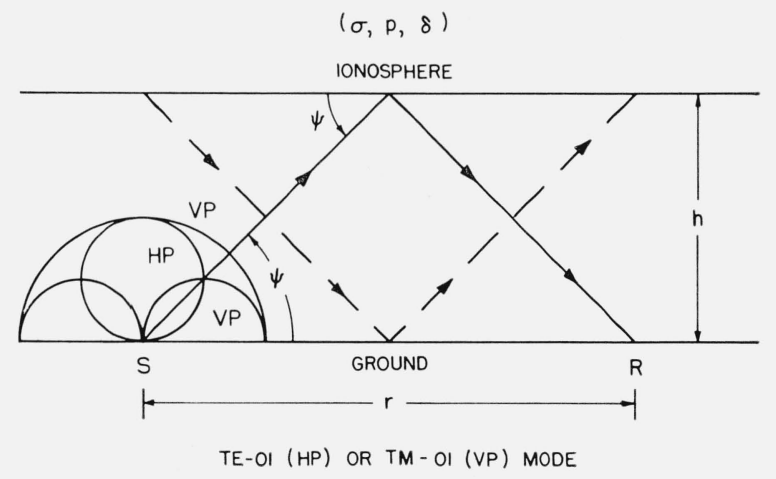

FIGURE 2. Theory of reflection for evaluation of transmission efficiency. 
In these formulas, the ionosphere is taken to have "pure conductivity," free of electricity; hence they are valid only if the displacement currents are much less than the conduction currents at any frequency under consideration.

It is noted that the ionosphere has a gradual transition of conductivity, rather than the definite boundary here assumed. This factor will be discussed further on.

Figure 2 shows incidentally the relations between the radiation patterns of a ground antenna $(S)$ and its coupling with the waveguide mode. 'These patterns are cross sections of the familiar "doughnut" pattern in various orientations. Each of these patterns is obtainable by a well-known simple antenna, such as a vertical wire, a raised horizontal wire, or a vertical loop with proper orientation.

The HP pattern is that of a vertical (axial) loop or a raised horizontal (transverse) wire. It shows zero radiation in the direction from $S$ to $R$. However, it shows substantial (less than maximum) radiation in the oblique direction of propagation of a component wave in the guide. Therefore it gives substantial coupling between the antenna and the TE-01 mode of horizontal polarization.

The elevation angle $(\psi)$ of oblique propagation in the waveguide is defined as follows:

$$
\sin \psi=\lambda / \lambda_{c} ; \cos \psi=\lambda / \lambda_{g} ; \tan \psi=\lambda_{g} / \lambda_{c}
$$

in which $\lambda_{g}$ is the so-called "guide wavelength."

As previously mentioned, the attenuation in propagation may be computed from the reflection loss, as determined by the elevation angle and the conductivity. This concept fails for the TM-00 mode, in which the elevation angle is zero, so it is not adequate for comparison of the three modes under consideration.

We return to the familiar viewpoint of waveguide modes, between conductive boundaries giving nearly complete reflection. The resulting attenuation can be computed from simple formulas, to be given. The relative attenuation in the three modes is graphed in figure 3 . The simplest mode (TM-00) is taken as a reference, because it propagates over the entire frequency range.

The following formulas give the attenuation in each of the three modes for the present configuration, in the limiting case of nearly complete reflection at the walls [Marcuvitz, 1951],

$\mathrm{TM}-00(\mathrm{VP}): \alpha_{0}=\frac{1}{4 \pi} \frac{2 \pi \delta}{\lambda_{c}} \frac{2 \pi r}{\lambda}$.

Here the height $(h)$ is replaced by $1 / 2$ wavelength at cutoff $\left(\frac{1}{2} \lambda_{c}\right)$ for the other two modes, although this mode has no cutoff.

$$
\begin{aligned}
\mathrm{TM}-01(\mathrm{VP}): \alpha_{v} & =\frac{1}{2 \pi} \frac{2 \pi \delta}{\lambda_{c}} \frac{2 \pi r}{\lambda} \frac{\lambda_{g}}{\lambda} \\
\alpha_{v} / \alpha_{0} & =2 / \cos \psi
\end{aligned}
$$

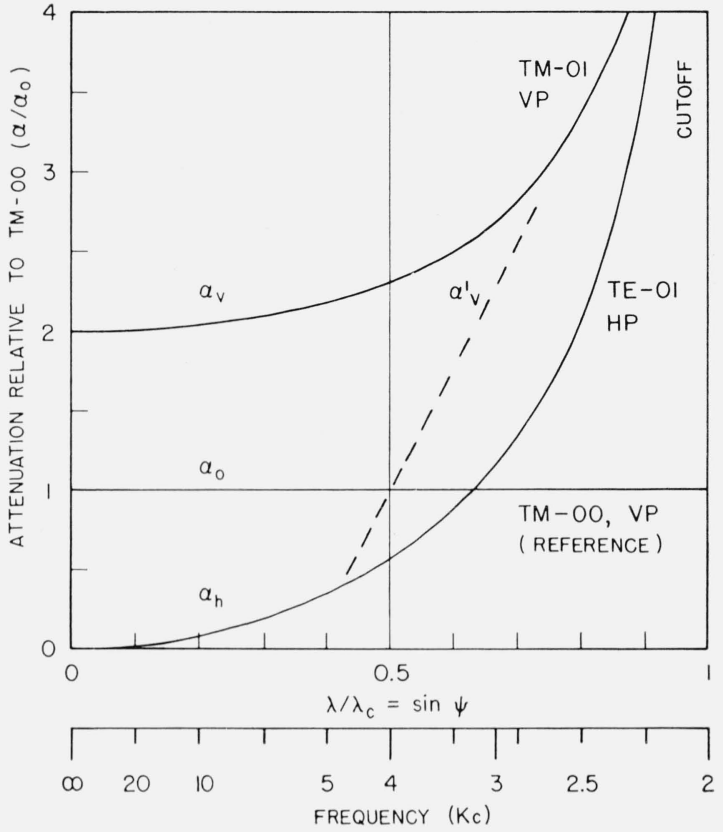

Figure 3. Relative altenuation of propagation in modes $T M-00, T M-01$, and TE-01.

$$
\begin{aligned}
\mathrm{TE}-01(\mathrm{HP}): & \alpha_{h}=\frac{1}{2 \pi} \frac{2 \pi \delta}{\lambda_{c}} \frac{2 \pi r}{\lambda_{c}} \frac{\lambda_{g}}{\lambda_{c}} \\
\alpha_{h} / \alpha_{0} & =2 \sin ^{2} \psi / \cos \psi=2 \sin \psi \tan \psi \\
\alpha_{h} / \alpha_{v} & =\left(\lambda / \lambda_{c}\right)^{2}=\sin ^{2} \psi .
\end{aligned}
$$

In these formulas $\alpha$ is the attenuation (napiers) for the radial distance $(r)$.

It is noted that this attenuation is only the component caused by dissipation in the upper boundary. This is added to the basic attenuation caused by radial divergence of the wave from sender to receiver. From the mode viewpoint in the present situation, this is a cylindrical wave guided between parallel plane conductors.

Referring to figure 3 , the horizontal scale is the wavelength ratio $\left(\lambda / \lambda_{c}\right)$ which is inversely proportional to frequency; this is chosen to present the entire range of propagation on a finite scale. Also it shows clearly the limiting conditions far from cutoff.

The two modes associated with oblique propagation have attenuation increasing toward the cutoff frequency. This may be explained by the increasing number of reflections in covering the distance.

The dissipation is caused by magnetic field at the upper boundary. Far from cutoff, the HP mode has the maximum concentration of magnetic field midway between the boundaries, so the dissipation is much the least of the three modes. This is analogous to the familiar TE-01 mode in circular waveguide. As in that case, the TE-01 mode between parallel planes has the least attenuation of all modes, far

\footnotetext{
2 This equation (9) is in agreement with a formula of J. R. Wait [1960].
} 
from cutoff. The marginal condition is

$$
\begin{array}{r}
\alpha_{h} / \alpha_{0}=1: \lambda / \lambda_{c}=\sin \psi=\lambda / \lambda_{c}=\sqrt{\frac{2}{\sqrt{17}+1}}=0.625 ; \\
f / f_{c}=\lambda_{c} / \lambda=1.60 .
\end{array}
$$

For the ionosphere, these relations are complicated by the fact that it may not behave as a "pure conductor," especially as it affects the TM-01 mode. The conduction current may be comparable with the displacement current. Therefore there is some difficulty in evaluating the apparent conductivity in the simple model here assumed.

In practical conditions, the curves in figure 3 fall short of representing the relative attenuation in the several modes. This happens from the failure of two assumptions: first, that the reflecting boundary is a good conductor; and second, that it has a step transition between the conductor and the wave medium. The former is to be discussed here; the latter at a point further on.

Continuing on the assumption of a step boundary, the relation shown between TM-00 and TE-01 modes is qualitatively valid for the usual conductivity of the ionosphere, at frequencies not too far from cutoff. On the other hand, the curve for the TM-01 mode $\left(\alpha_{v}\right)$ fails entirely for reasons associated with the angle of minimum reflection (Brewster angle). Far from cutoff (beyond the Brewster angle), at small angles approaching grazing incidence, the ionosphere behaves more like a magnetic wall (open-circuit) than an electric wall (short-circuit) for reasons not to be elaborated here. As a result, the waveguide propagation behaves more like the TM$0 \frac{1}{2}$ mode. The ionosphere becomes a much better reflector, so the attenuation becomes the least of all modes, approaching $1 / 4$ the attenuation of the TE-01 mode. The transition to this phenomenon is qualitatively indicated by the dashed line $\left(\alpha_{v}^{\prime}\right)$.

The location of the dashed line in figure 3 is indefinite because the relative attenuation varies with conditions. There is an experimental basis for specifying its intersection with unity, or the attenuation crossover of the TM-01 and TM-00 modes. It happens that VP measurements of atmospheric noise show a definite minimum at a certain frequency [C.C.I.R., 1957; Jean et al., 1961; Maxwell et al., $1963]$ and it may be inferred that one of these modes is predominant on either side of this frequency. The frequency of minimum noise is centered at 3 to $4 \mathrm{kc} / \mathrm{s}$, presumably nearer the higher value for conditions of lower ionosphere (height near $70 \mathrm{~km}$ ). Therefore $4 \mathrm{kc} / \mathrm{s}$ is here taken as a typical value of the crossover frequency, and the dashed line in figure 3 is so drawn.

From these considerations, figure 3 shows that the HP mode may have the least attenuation of all modes in the frequency range of about 3 to $5 \mathrm{kc} / \mathrm{s}$. At least, it is competitive with the two VP modes. This in itself is interesting and may not have been stated in earlier publications. However, there are other factors that give it a unique advantage, as will be explained.
For a stable transmission over a long range from one point to another, it is preferable to utilize only on waveguide mode. Figure 3 shows the two modes with vertical polarization, and furthermore shows that they are equal in attenuation at some frequency near $4 \mathrm{kc} / \mathrm{s}$. Diurnal variation of the ionosphere height will cause a variation of several cycles in their relative phase at the receiver. Therefore we have the possibility of slow periodic fading and occasional deep fading, in contrast to stable transmission.

On the other hand, there is only one mode with horizontal polarization and maximum amplitude. In the range of 3 to $5 \mathrm{kc} / \mathrm{s}$, we may consider the utilization of this TE- 01 mode to the exclusion of others. It has comparable attenuation, perhaps the lowest, and there would be no opportunity for fading. Therefore it may be the most useful mode for this frequency range. This concept is the subject of this paper. It will be considered further with reference to the practical problems of utilization.

The evaluation of attenuation in terms of skin depth $(\delta)$ is based on the concept of a wall of uniform high conductivity with a definite plane boundary. The gradual boundary of the ionosphere is better approximated by an exponential profile of conductivity, under study by the writer during the past 4 years [Wheeler, 1959, 1960, 1961, 1962] and more intensively by others [Wait and Walters, 1963]. There is a difference in behavior that can be described simply for horizontal polarization but not so simply for vertical polarization. The reasons for this will be given briefly.

In the usual "skin effect," the "hard" boundary of a good conductor acts as the boundary for both $E$ and $M$ fields. It causes appreciable loss associated with the $M$ field but no appreciable loss associated with the $E$ field. On the other hand, the "soft" boundary of the ionosphere may cause its effective level to appear at different heights, lower for the $E$ field than for the $M$ field [Wheeler, 1959, 1960, 1961]. This contributes a loss also associated with the $E$ field, which is comparable with that of the $M$ field. In the actual profile of the ionosphere, the lower level of the $E$-field boundary is usually in a region of more gradual variation, so the $E$-field loss is likely to be the greater of the two components.

With vertical polarization, both $E$ and $M$ fields are maximum at a conductive boundary, so both are subject to the losses of a "soft" boundary. With horizontal polarization, however, the $M$ field is maximum and the $E$ field is minimum at a conductive boundary, so only the $M$-field losses are appreciable. This enables a simple description of the boundary reflection for horizontal polarization [Wait and Walters, 1963].

In the TE-01 mode, the boundary losses can be computed by making the following substitution in formula (7). The effective skin depth appears to be

$$
\delta=\pi h_{1}
$$

in which $h_{1}$ is the "napier height" in the exponential profile, that is, the change of height in which the 
conductivity increases in the ratio of $e$, the base of natural logarithms. (This rule was developed by the writer in 1960.) In other words,

$$
\frac{\text { conductivity at } h+\Delta h}{\text { conductivity at } h}=\exp \Delta h / h_{1} \text {. }
$$

In the usual skin effect, the skin depth is proportional to the $1 / 2$ power of wavelength $(\delta \propto \sqrt{\lambda})$. On the other hand, in the exponential profile, the apparent skin depth is invariant with wavelength. This implies that the reflection takes place at different levels of conductivity, proportional to wavelength, so the usual variation with wavalength is compensated.

Ref erring to figure 3 , if the skin depth is constant, the attenuation in the reference mode TM-00 varies inversely with wavelength or directly with frequency. Either side of the crossover of the two VP modes, one or the other has decreasing attenuation. This is consistent with the concept that the VP noise spectrum is subject to maximum attenuation at the crossover.

In figure 3 , the extra $E$-field loss in VP is ignored. It is expected that the relative attenuation of the HP mode will be found to be much less, perhaps down to $1 / 3$ the amount indicated by the curves. If so, this will yield a great advantage for HP over $\mathrm{VP}$ in the frequency range shown around $4 \mathrm{kc} / \mathrm{s}$. The attenuation rate for the VP modes has been reviewed recently [Maxwell et al., 1963] and the resulting values in the frequency range are much graater than would be expected for HP [Wait and Walters, 1963].

Here we should be reminded that the VLF sending station has to compete with a high level of atmospheric noise from lightning. There is a need for tests of the HP noise spectrum, since only the VP spectrum has been reported. Horizontal lightning strokes are presumably more frequent than vertical, and have a height advantage over an HP antenna near the ground. They are not expected to have any minimum of spectral density, since the crossover phenomenon is avoided in HP. These factors suggest that HP may be subject to a handicap in the noise level to be surmounted by radiation of power from the sending antenna.

It has been assumed that higher modes have much greater attenuation, and therefore are relatively too weak to be useful or to cause appreciable cancellation. The justification is very simple. We may compare the " 01 " mode with the " $0 N$ " mode of higher order $(N>1)$. First we note that the cutoff wavelength is $1 / N$ as great, so the angle of elevation $(\psi)$ becomes about $N$ times as great; this causes about $N$ times the loss in one reflection. (The reflection loss approaches zero at grazing incidence.) Second, this means about $N$ times the number of reflections for the same distance. We conclude that the rate of attenuation in the " $0 N$ " mode is about $N^{2}$ times as great as that in the "01" mode. This comparison applies similarly to the TM and TE modes in the present discussion.
With vertical polarization, the imperfect conductivity of the ground contributes appreciable attenuation, greater at higher frequency. This effect is severe in case of dry ground, especially mountains or ice cap. On the other hand, with horizontal polarization, this component of attenuation is less in general; in particular it is less at higher frequency and is negligible in comparison with the ionosphere component.

For any particular mode in a waveguide, there is a coupling factor associated with any type of antenna. This coupling may be evaluated as complete (1) or partial but useful $(<1)$ or nominally zero $(0)$. In these terms, table 1 gives the rating of various types of antennas with respect to the three modes of interest.

TABLE 1. Mode coupling of various types of antennas

\begin{tabular}{|c|c|c|c|}
\hline \multirow{2}{*}{ Antenna (at ground) } & \multicolumn{3}{|c|}{ Mode coupling } \\
\hline & $\begin{array}{c}\text { VP } \\
\text { TM-00 }\end{array}$ & $\begin{array}{c}\text { VP } \\
\text { TM-01 }\end{array}$ & $\underset{\text { TE-01 }}{H P}$ \\
\hline $\begin{array}{l}\text { Omnidirective: } \\
\text { (1) Vertical wire } \\
\text { (2) Horizontal wire, crossed pair } \\
\text { (3) Vertical loop, crossed pair }\end{array}$ & $\begin{array}{l}1 \\
0 \\
1\end{array}$ & $\begin{array}{r}<1 \\
<1 \\
1\end{array}$ & $\begin{array}{r}0 \\
<1 \\
<1\end{array}$ \\
\hline $\begin{array}{l}\text { Directive: } \\
\text { (4) Oblique wire, end direction } \\
\text { (5) Horizontal wire, end direction } \\
\text { (6) Horizontal wire, side direction } \\
\text { (7) Vertical loop, planar direction } \\
\text { (8) Vertical loop, axial direction }\end{array}$ & $\begin{array}{r}<1 \\
0 \\
0 \\
1 \\
0\end{array}$ & $\begin{array}{r}0 \\
<1 \\
0 \\
1 \\
0\end{array}$ & $\begin{array}{r}0 \\
0 \\
<1 \\
0 \\
<1\end{array}$ \\
\hline
\end{tabular}

Notes:

(2) (3) Rotary phase; can use diversity with opposite rotation.

(4) Designed to cancel T M-01 mode in one direction at one frequency.

(4) (5) (6) (8) Selection of single mode, effective in only one direction.

(1) and (2) Sending-receiving combination for selection of TM-01 mode, both omnidirective.

(2) and (7) Sending-receiving combination for selection of TM-01 mode,

one omnidirective and the other directive; can use

one omnidirective and the
(7) for direction finding.

(1) and (7) Sending-receiving combination for both VP modes, the usual combination; can use (7) for direction finding.

Figure 4 gives some curves of the variation of mode coupling with wavelength or frequency. As in figure 3 , these are referred to the TM-00 mode, and the same horizontal scale is used. These are based on the vertical loop antenna, being the only type that couples with all three modes.

The following formulas give the mode coupling ratios on the assumption that the antenna is a small horizontal magnetic dipole (vertical loop) located on or near the lower boundary (ground).

$\frac{\mathrm{TM}-01(\mathrm{VP})}{\mathrm{TM}-00(\mathrm{VP})}: k_{v}=\sqrt{\frac{2}{\cos \psi}}$

$\frac{\mathrm{TE}-01(\mathrm{HP})}{\mathrm{TM}-00(\mathrm{VP})}: k_{h}=\sin \psi \sqrt{\frac{2}{\cos \psi}}=\sqrt{2 \sin \psi \tan \psi}$

in which $k$ is the mode-coupling voltage or current ratio referred to the TM-00 (TEM) mode.

This ratio may be evaluated for a receiving loop, by comparing the values of induced voltage from plane waves of two modes, carrying equal values of power. In general, the mode coupling depends 


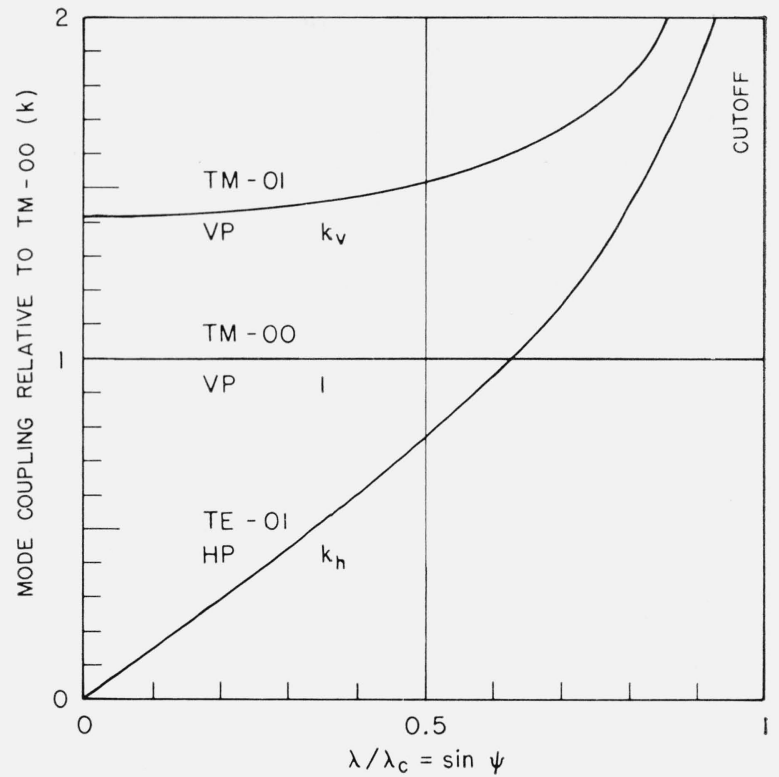

Figure 4. Relative mode coupling between loop and modes $T M-00, T M-01$, and $T E-01$.

on the factors shown in figure 2, namely, the elevation angle $(\psi)$ and the antenna radiation pattern. For VP, the vertical loop has a pattern invariant with angle, so the coupling $\left(k_{v}\right)$ includes only the waveguide effect (in terms of $\cos \psi$ ). For $\mathrm{HP}$, the pattern contributes an extra factor $(\sin \psi)$, which is of particular interest in the present discussion.

The mode coupling ratios are simply related with the attenuation ratios, as will be seen by comparing (6) and (8) with (13) and (14). This results from the fact that they both depend on the magnetic field adjacent to the boundaries.

In figure 4 , the mode coupling ratio $\left(k_{v}\right)$ between the two VP modes is nearly constant, except near cutoff, and is not far from unity. On the other hand, the HP mode coupling $\left(k_{h}\right)$ varies more than proportional to wavelength. This is because the coupling increases with the elevation angle $(\psi)$, as previously mentioned with reference to figure 2 .

In the range of 3 to $5 \mathrm{kc} / \mathrm{s}$, of particular significance in figure 3 , the coupling of the HP mode is somewhat less than that of the VP modes. However, this slight deficiency is present only at each end of the circuit; over a long distance, it is easily offset by a slightly lower rate of attenuation, or by other advantages such as stability against fading.

Referring to figure 4, the mode coupling of the loop may be compared with that of a straight wire. A vertical wire couples only with the VP modes; the coupling with TM-01 decreases toward zero at cutoff. A horizontal wire couples with TM-01 and TE-01 modes, not with TM-00; its coupling with TM-01 or TE-01 has the same variation as $k_{h}$ in figure 4.

Referring to table 1 , there are tabulated the modecoupling ratings of eight types of antennas that may be interesting with respect either to omnidirective coverage or to unique properties in one direction. The table is self-explanatory, so the discussion will be limited to some relations of particular interest.

The vertical wire is the only antenna that couples with only VP, not $\mathrm{HP}$ in any direction. The horizontal wire or the vertical loop couples with both VP and HP; the loop couples with all three modes. These two types have directive properties that enable the selection of either VP or HP, to the exclusion of the other, in some direction.

It is concluded that the single-mode feature of $\mathrm{HP}$ can be assured only in a point-to-point circuit, having one or both antennas of type (6) or (8). Each of these couples only HP in one direction.

\section{Antennas}

The utilization of any particular mode of propagation requires the selection of types of antennas which are suited for coupling with the mode. In the present discussion, the use of horizontal polarization instead of the usual vertical polarization requires coupling with the TE-01 mode instead of the usual TM-01 or TM-00 modes. The introduction to the mode in figure 1 shows the common loop antenna used in different orientations relative to the direction of transmission from sander $(S)$ to receiver $\left(R\right.$ or $\left.R^{\prime}\right)$.

Figure 5 shows the horizontal wire (a) and the vertical loop (b), which are alternative types for utilizing the single-mode feature of HP propagation in a direction perpendicular to the vertical plane of the antenna. Each will be described, for the case of a "small" antenna, having effective length $(l)$ and effective height $\left(l^{\prime}\right)$ less than $1 / 8$ wavelength. The simple structures to be shown are typical of sending antennas of rather large size for radiation of high power.

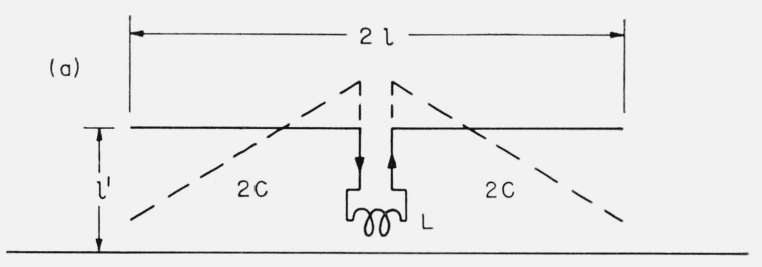

(b)

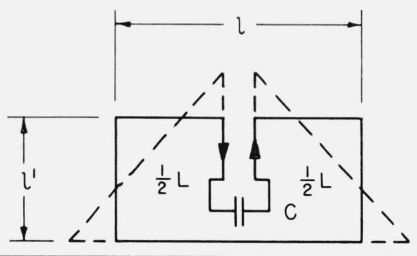

Figure 5. Alternative sending antennas on the ground.

(a) Horizontal electric dipole (horizontal wire).

(b) Alternative sending antennas on the ground. 
Figure 5(a) shows a horizontal wire which behaves as a horizontal electric dipole of a certain effective length $(l)$ and effective height $\left(l^{\prime}\right)$. It has a balanced coupling with the circuit, so the effective capacitance $(C)$ is that of the two halves in series. It is tuned to resonance by an inductor $(L)$.

Figure 5(b) shows a vertical loop which behaves as a horizontal magnetic dipole of a certain effective area $\left(l \times l^{\prime}\right)$. It has a balanced coupling with tha circuit, so the effective inductance $(L)$ is that of the two halves in series. It is tuned to resonance by a capacitor $(C)$

In figure $5(\mathrm{a})$ or $5(\mathrm{~b})$, the HP mode coupling is determined by the effective area $\left(l \times l^{\prime}\right)$. The top wire may be horizontal (solid lines) or sloping (dashed lines). The latter offers a particular advantage for the horizontal wire (a) because the current is greater near the center; as a result, the center has to be raised only half as much as the ends are lowered, so the average height can be decreased. In either case, the sloping form enables the use of a single tower.

For omnidirective coverage, either type can be utilized in a crossed pair having quadrature relative phase of coupling with the circuit. The sloping form enables the use of a single tower for both antennas of the pair.

As in any capacitive antenna, the use of grounded metal supporting structures causes a reduction of effective height of the horizontal wire (a). In an inductive antenna, such as the loop (b), a thin vertical tower has no appreciable effect; the use of a thick metal tower or grounded guy wires may cause reduction of effective height, but less than the effect on a capacitive antenna.

As in "small" sending antennas in general, the radiation of high power requires a higher voltage on the capacitive type (a) and a higher current on the inductive type (b). This essential difference and other practical considerations influence the choice between these two types [Wheeler, 1958a].

A receiving antenna may be much smaller, for two reasons. First, there is no requirement for handling high power. Second, a rather small size is sufficient to receive atmospheric noise at a level higher than circuit noise. There is more freedom of design in the smaller size.

A small electric dipole has two practical limitations. First, the reactance becomes so high as to impose difficulties in circuit design, and there is no way of transforming the reactance to a lower value in the antenna. Second, there is no core material available for enabling a further reduction in size while retaining the same efficiency.

A small magnetic dipole or loop offers advantages in both of these respects. First, while the reactance of one turn is low, it may be transformed to a convenient value by using a number of turns. Second, a ferrite core enables a further reduction in size while retaining the same efficiency. These and other practical considerations lead to the use of a small loop for reception, especially for submarine reception while submerged [Wheeler, 1958b].
The radiation resistance of an antenna under the ionosphere is essentially different from that in free space. It is seldom formulated, because there is no practical difference in the usual VLF band (10 to 30 $\mathrm{kc} / \mathrm{s}$ ), such that the ionosphere is more than two wavelengths from the antenna.

At lower frequencies, nearer to cutoff in the waveguide between ionosphere and ground, there is a need for restating the radiation resistance. The ionosphere has more effect on the resistance. Furthermore, the power radiated in each mode can be separately identified with a value of radiation resistance. 'This enables an evaluation of the efficiency of radiation into the mode to be utilized.

The resistance representing radiation into the TE-01 mode, in either of the types shown in figure 5, is given by the following formula, derived from familiar waveguide principles,

$$
\begin{aligned}
R & =\frac{\pi}{4} R_{c}\left(\frac{2 \pi l}{\lambda}\right)^{2}\left(\frac{l^{\prime}}{h}\right)^{2} \frac{\lambda_{g}}{\lambda_{c}}=\frac{1}{4 \pi} R_{c}\left(\frac{2 \pi l}{\lambda_{c}}\right)^{2}\left(\frac{2 \pi l^{\prime}}{\lambda_{c}}\right)^{2} \frac{\lambda_{c}}{\lambda} \frac{\lambda_{g}}{\lambda} \\
& =\frac{1}{2 \pi} R_{c}\left(\frac{2 \pi l}{\lambda_{c}}\right)^{2}\left(\frac{2 \pi l^{\prime}}{\lambda_{c}}\right)^{2} \frac{1}{\sin 2 \psi}
\end{aligned}
$$

The variation with frequency becomes very simple. There is a minimum value for the condition,

$$
f / f_{c}=\sqrt{2} ; \psi=\pi / 4 ; \sin 2 \psi=1 .
$$

In the waveguide modes under the ionosphere, its effective height is involved directly $(h)$ or indirectly in terms of the resulting cutoff wavelength $\left(\lambda_{c}\right)$. This cylindrical wave is an essential distinction from the spherical wave in free space, especially in the usual cases where one mode predominates over the others.

The efficiency of radiation into this mode is the ratio of $R$ over the total resistance. The latter includes not only the heat losses in the antenna and ground and the rest of the tuned circuit, but also the radiation into any other propagating modes. High efficiency of radiation into one mode requires the exclusion of coupling with any other propagating modes.

The antennas of figure 5 couple not only with the TE-01 mode of HP propagation, but also with one or both of the VP modes (in other directions). Therefore a high efficiency of radiation in the TE-01 mode is impossible. In practice, however, the heat losses are usually sufficient to preclude high efficiency, so the extra radiation resistance of other modes is a minor handicap.

\section{Transmission Efficiency}

The transmission circuit from sender to receiver should be evaluated with reference to all significant factors. Some of these are elusive but generally recognized so they need not be elaborated here. A few that are particularly relevant or unusual will be described with reference to a computed example. 
Table 2 outlines a set of conditions and various factors resulting therefrom. It is based on a pointto-point transmission circuit relying on horizontal polarization in the TE-01 mode under the ionosphere. The natural conditions are given values that are typical to the extent of present knowledge based directly or indirectly on experimental evidence. The operating conditions are selected to bring out the capabilities of horizontal polarization. The table is self-explanatory, as a basis for some comments.

The frequency $(4 \mathrm{kc} / \mathrm{s})$ is approximately that at which horizontal polarization appears to offer the greatest advantage over vertical polarization. It is double the cutoff frequency $(2 \mathrm{kc} / \mathrm{s})$ based on the ionosphere height.

The attenuation by radial divergence of a cylindrical wave is given by the power ratio,

$$
\frac{\lambda / 2 \pi}{2 \pi r}
$$

No attempt is made to refine this ratio in respect to the guide wavelength and any directivity that may be utilized.

The least is known about the height interval of transition from dielectric to conductor in the ionosphere serving as the upper boundary of a waveguide. This is expressed in terms of the "napier height" related to the most recent publication of Wait and et al. [1963]. The value assumed $(2 \mathrm{~km})$ is said by him to be typical of daytime ionosphere, in a discussion directed to horizontal and vertical polarization at frequencies in the VLF band. The reflection coefficient is computed from his formula, and is related to the effective skin depth as discussed herein. This leads to the attenuation caused by loss in reflection at the ionosphere.

TABLE 2. Transmission circuit using horizontal polarization

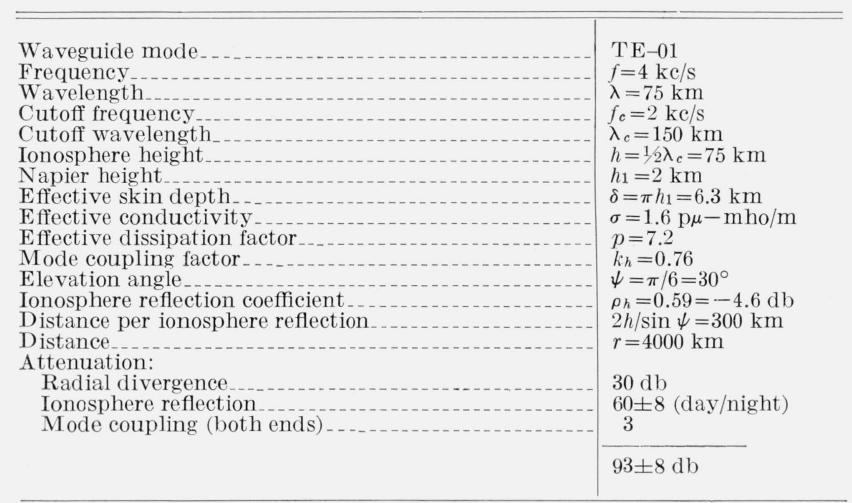

The diurnal variation of attenuation is even more uncertain. The value given is estimated on the basis of some experimental evidence for vertical polarization [Maxwell et al., 1963] and modified for the expected improvement in stability for horizontal polarization.

The loss in mode coupling at both ends of the circuit is evaluated for the TE- 01 mode relative to the TM-00. It becomes a minor factor in this example.

The three kinds of attenuation included in this outline emphasize the peculiarities of this mode. It is notable that the major factor is the attenuation caused by incomplete reflection from the ionosphere, even though this is much less for horizontal polarization. These components of attenuation add up to about $100 \mathrm{db}$ for a distance of $4,000 \mathrm{~km}$. This is typical of the greatest distance over which the TE01 mode may be useful.

An implicit factor in reaching this conclusion is the noise level from lightning. 'This is an unknown for horizontal polarization, but the receiving antenna responds also to waves of vertical polarization arriving from other directions. The rate of attenuation in both polarizations discriminates against noise sources at great distances, so the noise level far from the equator is much lower than usually experienced in the active VLF range of 10 to $30 \mathrm{kc} / \mathrm{s}$.

\section{Conclusion}

It appears that there is a part of the frequency spectrum that may be best utilized in the TE-01 waveguide mode under the ionosphere, characterized by horizontal polarization. Some of the advantages and other peculiarities of this mode may be summarized as follows.

(1) In a frequency range near $4 \mathrm{kc} / \mathrm{s}$, this mode promises the lowest rate of attenuation of all modes under the ionosphere, as in the familiar TE-01 mode in circular waveguide. In comparison with the closest competitors, the TM-00 and TM-01 modes characterized by vertical polarization, it is expected to offer about half the rate of attenuation. Its cutoff frequency is near $2 \mathrm{kc} / \mathrm{s}$, and its advantage appears at somewhat higher frequencies.

(2) The mechanism of reflection at the ionosphere is simpler for horizontal polarization, involving mainly the magnetic field rather than both electric and magnetic fields. It is expected that there will be less variation of attenuation with changing conditions (diurnal cycles, disturbances, etc.). This advantage is particularly noticeable in the theory and operation of the "soft" boundary prevalent below the ionosphere, as distinguished from the "hard" boundary previously assumed as a simple model.

(3) There are some antenna configurations suited for coupling with the TE-01 mode. The coupling is somewhat less than that available for vertical polarization, but this small handicap is negligible in comparison with the gain by the lower rate of attenuation.

(4) In a point-to-point transmission circuit, there are simple types of antennas that select $\mathrm{HP}$ modes to the exclusion of VP modes. Since the TE-01 mode has much the lowest attenuation of all $\mathrm{HP}$ modes, this feature can be used to assure single-mode propagation. This is the most stable condition in a waveguide because there is no possibility of fading caused by interference between two modes. One 
or both of the antennas should be a horizontal wire above the ground or a vertical loop, in a plane perpendicular to the direction of the transmission path. The former is particularly suited for the sending end, the latter for the receiving end.

(5) The loss on reflection from the ground is the least for horizontal polarization, being much less than the loss on reflection from the ionosphere. Therefore there is no appreciable handicap in transmission over ground of low conductivity, such as mountains or ice cap.

(6) The noise level for HP has not been thoroughly measured and reported. In the subject frequency range, the moderately high attenuation reduces the contribution from noise sources at a long distance. This is particularly true of VP noise, to which any receiving antenna is likely to be responsive in some directions.

(7) It happens that there is no simple antenna, for location just above the ground, which will select HP to the exclusion of VP in all directions. Therefore we cannot secure omnidirective coverage in both transmission and reception with the full advantage of HP. This is not an appreciable handicap at any frequency for which the HP mode has much the lowest rate of attenuation. This factor does preclude the use of HP for accurate direction finding, because a loop antenna would be confused by a mixture of HP and VP. Perhaps this last property could be used intentionally to confuse the direction finding attempts of an enemy.

(8) The use of frequencies near $4 \mathrm{kc} / \mathrm{s}$, in contrast to the lowest frequencies now in use $(14-20 \mathrm{kc} / \mathrm{s})$, would reduce the attenuation rate in sea water to about one-half. This is an obvious advantage in communication to a submerged submarine, but must be weighed in the context of the other significant factors.

A computed example indicates that the subject mode should be useful for communication over a distance of the order of $4,000 \mathrm{~km}$.

It is submitted that this mode offers the most effective utilization of a certain part of the frequency spectrum, in transmission under the ionosphere. Therefore further theoretical and experimental studies are recommended.

The present understanding of mode propagation under the ionosphere is attributed to J. R. Wait, and has been relied on as the background for this presentation. It is an application of the waveguide principles that are familiar in the field of microwaves, as most intensively developed at the M.I.T. Radiation Laboratory during: World War II.

To the National Bureau of Standards, and particularly to the Staff at Boulder, Colo., we are all most deeply indebted for their studies of radio noise. Here we have relied on the more specialized tests of the noise spectrum, reported by A. D. Watt, E. L. Maxwell, A. G. Jean, and their associates.

\section{References}

Comite Consutatif Internationa Radio (C.C.I.R.) (1957), Revision of atmospheric radio noise data, Report No. 65, International Telecommunication Union, Geneva $(10 \mathrm{kc} / \mathrm{s}$ to $100 \mathrm{Mc} / \mathrm{s}$ ).

Jean, A. G., A. C. Murphy, J. R. Wait, and D. F. Wasmundt (Sept.-Oct. 1961), Observed attenuation rate of ELF radio waves, J. Res. NBS 65D (Radio Prop.), No. 5, 475-479 $(50-400 \mathrm{c} / \mathrm{s})$.

Marcuvitz, N. (1951), Waveguide Handbook (Attenuation in rectangular waveguides, Rad. Lab. Series, 10, 56-66 (McGraw-Hill Book Co., Inc. New York, N.Y.).

Wait, J. R. (Mar.-Apr. 1960), Terrestrial propagation of very-low-frequency radio waves. A theoretical investigation, J. Res. NBS 64D (Radio Prop.), No. 2, 153-205.

Wait, J. R., and I. C. Walters (May-June 1963), Reflection of VLF radio waves from an inhomogeneous ionosphere. Part I. Exponentially varying isotropic model, J. Res. NBS 6\%D (Radio Prop.) No. 3, 361-367.

Maxwell, E. L., and D. L. Stone (May 1963), Natural noise fields from 1 eps to $100 \mathrm{kc} / \mathrm{s}$, Trans. IEEE AP-11, 339-343. (Maximum attenuation $17-25 \mathrm{db} / 1000 \mathrm{~km}$ at $3 \mathrm{kc} / \mathrm{s}$ ).

Wheeler, H. A. (Jan. 1958a), Fundamental relations in the design of a VLF transmitting antenna, Trans. IRE AP-6, $120-122$.

Wheeler, H. A. (Jan. 1958b), Fundamental limitations of a small VLF antenna for submarines, Trans. IRE AP-6, $123-125$.

Wheeler, H. A. (Sept. 1959), UFL antennas and propagation (Ionosphere with exponential profile of conductivity), private communications.

Wheeler, H. A. (Dec. 1960), ULF deep waveguide-notes (Rules for $\mathrm{E}$ and $\mathrm{M}$ boundaries of thermal ionosphere, exponential profile of conductivity), private communications.

Wheeler, H. A. (Mar.-Apr. 1961), Radio wave propagation in the Earth's crust, J. Res. NBS 65D (Radio Prop.), No. 2, 189-191. (Concept of E and M boundaries in thermal ionosphere with exponential profile of conductivity.)

Wheeler, H. A. (May 1962), Electromagnetics and communications, the low frontier, Proc. IRE 50, 582-583. (Proposal of TE-01 mode of horizontal polarization under ionosphere, around $3 \mathrm{kc} / \mathrm{s}$.)

\section{Additional References}

Crichlow, W. Q., D. F. Smith, R. N. Morton, and W. R. Corliss (Aug. 1955), Worldwide radio noise levels expected in the frequency band $10 \mathrm{kc} / \mathrm{s}$ to $100 \mathrm{Mc} / \mathrm{s}$, NBS Circ. 557.

Galejs, J. (May-June 1961), Excitation of VLF and ELF radio waves by a horizontal magnetic dipole (emphasis on vertical polarization), J. Res. NBS 65D (Radio Prop.), No. 3, 305-311.

Wait, J. R. (June 1957), The attenuation versus frequency characteristics of VLF radio waves (computed for modes 0,1 , ete.), Proc. IRE 45, 768-771.

Wait, J. R. (May 1963), The possibility of guided electromagnetic waves in the Earth's crust, Trans. IEEE AP-11, 330-335. (Exponential profile of conductivity at thermal ionosphere.)

Watt, A. D., and E. L. Maxwell (June 1957), Characteristics of atmospheric noise from 1 to $100 \mathrm{kc} / \mathrm{s}$, Proc. IRE 45, 787-794. (Maximum attenuation at 3-4 kc/s, vertical polarization.)

Wheeler, H. A. (Apr. 1948), Radiation resistance of an antenna in an infinite array or waveguide, Proc. IRE 36, $479-487$.

Wheeler, H. A. (Nov. 1952), Universal skin-effect chart for conducting materials, Electronics, 25, No. 11, 152-154. 